\title{
Seventh-order wavefront modulation with a gravity-neutral optofluidic deformable phase plate
}

\author{
Pouya Rajaeipour $\odot$, a,b,* Martin Sauther, ${ }^{\text {a }}$ Kaustubh Banerjee $\odot,{ }^{\text {a,b }}$ \\ Hans Zappe $\odot,{ }^{a}$ and Çağlar Ataman $\odot^{a}$ \\ ${ }^{a}$ University of Freiburg, Department of Microsystems Engineering, Gisela and Erwin Sick \\ Laboratory for Micro-optics, Freiburg, Germany \\ ${ }^{b}$ Phaseform GmbH, Freiburg, Germany
}

\begin{abstract}
A 63-electrode optofluidic refractive wavefront modulator enabling spatial frequency correction up to the seventh radial Zernike order, the highest spatial order ever achieved with a refractive dynamic wavefront modulator, is presented. The modulator is designed so that aberration correction performance is nearly identical in both horizontal and vertical orientations, virtually eliminating the gravity-induced parasitic surface deformations typical for optofluidic devices. With 55 of its electrodes located within the $1-\mathrm{cm}$ clear pupil, the modulator offers the versatility of continuous face-sheet deformable mirrors within a compact, high efficiency, and transmissive device. Using a fluidic interfacing method based on wafer-level 3D microstructuring of glass, the modulator is only 0.86-mm-thick, facilitating the cascading of multiple modulators within close proximity. We demonstrate a bi-directional stroke of more than $13 \lambda$, and replications of Zernike mode shapes up to the seventh radial order with high fidelity, representing a significant leap in the performance of ultra-miniaturized refractive wavefront modulators. () The Authors. Published by SPIE under a Creative Commons Attribution 4.0 Unported License. Distribution or reproduction of this work in whole or in part requires full attribution of the original publication, including its DOI. [DOI: 10.1117/1.JOM.1.3.034502]
\end{abstract}

Keywords: adaptive optics; phase modulation; aberration correction; optofluidics; refractive; gravity-neutral.

Paper 21009 received May 4, 2021; accepted for publication Jul. 15, 2021; published online Aug. 5, 2021.

\section{Introduction}

Wavefront modulators are key components for adaptive optics (AO) systems, allowing dynamic correction of optical aberrations induced by the refractive index inhomogeneity of the imaging medium and/or the specimen, and the imperfections of an optical system. ${ }^{1}$ They have found a wide variety of applications in ground-based telescopes, ${ }^{2}$ biomedical imaging, ${ }^{3-5}$ vision science,,${ }^{6,7}$ free-space optical communication, ${ }^{8}$ and laser processing. ${ }^{9}$ The majority of these applications rely on deformable mirrors (DMs) for spatial light modulation since these combine high efficiency, wavelength, and polarization independence, with good articulation (e.g., high-order aberration correction). Integration of DMs into high-end optical systems, however, is hindered by the need for the additional hardware complexity of folded optical paths. For emerging AO concepts, such as multi-conjugate or woofer-tweeter AO, which use multiple wavefront modulators either in close succession or at conjugate planes, the use of DMs becomes practically infeasible for more than two modulators..$^{10-12}$

We have recently demonstrated the concept of an optofluidic refractive wavefront modulator, the deformable phase plate (DPP), which offers the versatility of reflective DMs in the form of a more practical compact refractive device ${ }^{13,14}$ which thus also enables the use of one or more adaptive elements in the beam path of a single optical system. ${ }^{15,16}$ In contrast to other refractive wavefront modulators based on tunable or adaptive lenses, ${ }^{17-20}$ the DPP features a 2D array of transparent electrostatic actuators within its clear pupil and was shown to enable aberration

*Address all correspondence to Pouya Rajaeipour, pouya.rajaeipour@imtek.uni-freiburg.de 
correction up to fifth radial Zernike order with a peak-to-valley wavefront modulation of up to $15 \lambda$ in the visible range.

Presented here is an advanced implementation of the DPP, which using 63 electrodes and a sophisticated optofluidic design, can replicate up to the seventh radial Zernike modes in both horizontal and vertical orientations with negligible deviations in device performance due to gravity effects. Fabricated using an innovative process for wafer-level 3D micro-structuring of glass, the fluidic interfaces of the DPP are embedded into a 0.8-mm-thick glass substrate, limiting the total device thickness to $0.86 \mathrm{~mm}$. As a result, multiple DPPs may easily be cascaded for enhanced correction performance ${ }^{16}$ or multi-conjugate AO. ${ }^{10}$ The combination of its compact form factor, compatible with the 30-mm optomechanical cage systems commonly used in optics and photonics laboratories, high spatial frequency correction capability and polarization independent refractive operation makes the DPP an ideal solution for many contemporary and future $\mathrm{AO}$ and beam shaping applications.

\section{Concept}

Figure 1 shows different working regimes of available wavefront modulators. They are categorized based on the typical number of spatial mode shapes (such as Zernike modes) that describe their wavefront modulation capability. For these modulators, the degrees of freedom is defined based on their number of actuators or pixels in the case of liquid-crystal spatial light modulators (LC-SLMs). The range starts from focus tuning devices requiring only one degree of freedom, all the way to addressing millions of spatial frequencies that are only realized with pixelated LC-SLMs. While for almost all the spectrum, there are state-of-the-art reflective solutions, the majority of the available refractive wavefront modulators are graded for the low spatial order wavefront modulation. Furthermore, the transmissive LC-SLMs are polarization dependent and suffer from diffraction losses. ${ }^{21}$ The optofluidic wavefront modulator developed in this study is intended for addressing this gap by providing a similar scalability and articulation to that of the continuous face sheet DMs but in transmission mode.

Figure 2(a) shows the schematic top and cross-sectional views of the developed electrostatically actuated optofluidic wavefront modulator. Its structure comprises a polymeric deformable membrane suspended over a micro-machined ring spacer on a glass substrate, forming an optofluidic chamber. The chamber is filled with an optical liquid using fluidic tubes that are inserted in the through-glass channels. Figure 2(b) shows the working principle of the DPP. 63 transparent electrodes are embedded on the glass substrate in the form of a highly dense 2D array. Each electrode is individually addressable via the contact pads that circumscribe the membrane supporting spacer. The membrane is also conductive and is held at ground potential with respect to the electrode array. Deforming the membrane's surface by applying voltage to any combination of the actuators results in the displacement of the liquid volume, manipulating the transmissive optical path, and resulting in voltage-dependent wavefront modulation of light. While electrostatic actuation allows for a pull-only (attractive) operation, due to the

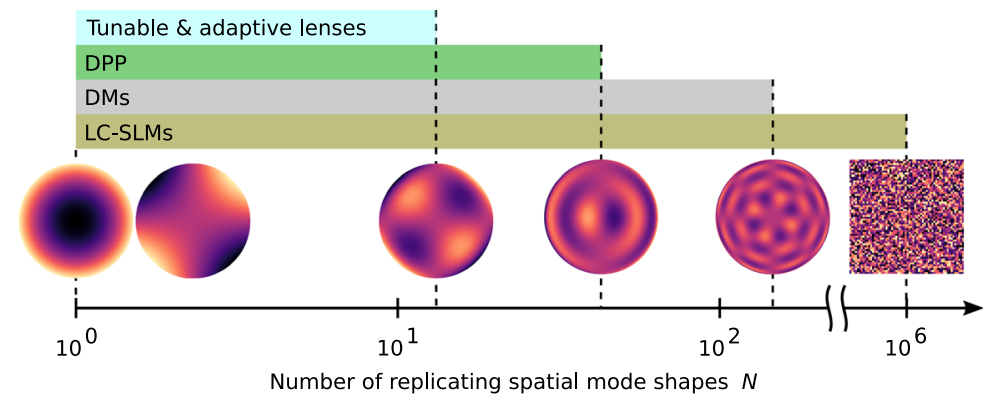

Fig. 1 Different working regimes of available wavefront modulators, categorized with respect to the required number of spatial mode shapes that describe their modulation capability. The colored bars depict the type of wavefront modulators; DM: deformable mirror, LC-SLM: liquid crystal spatial light modulator, DPP: deformable phase plate. 


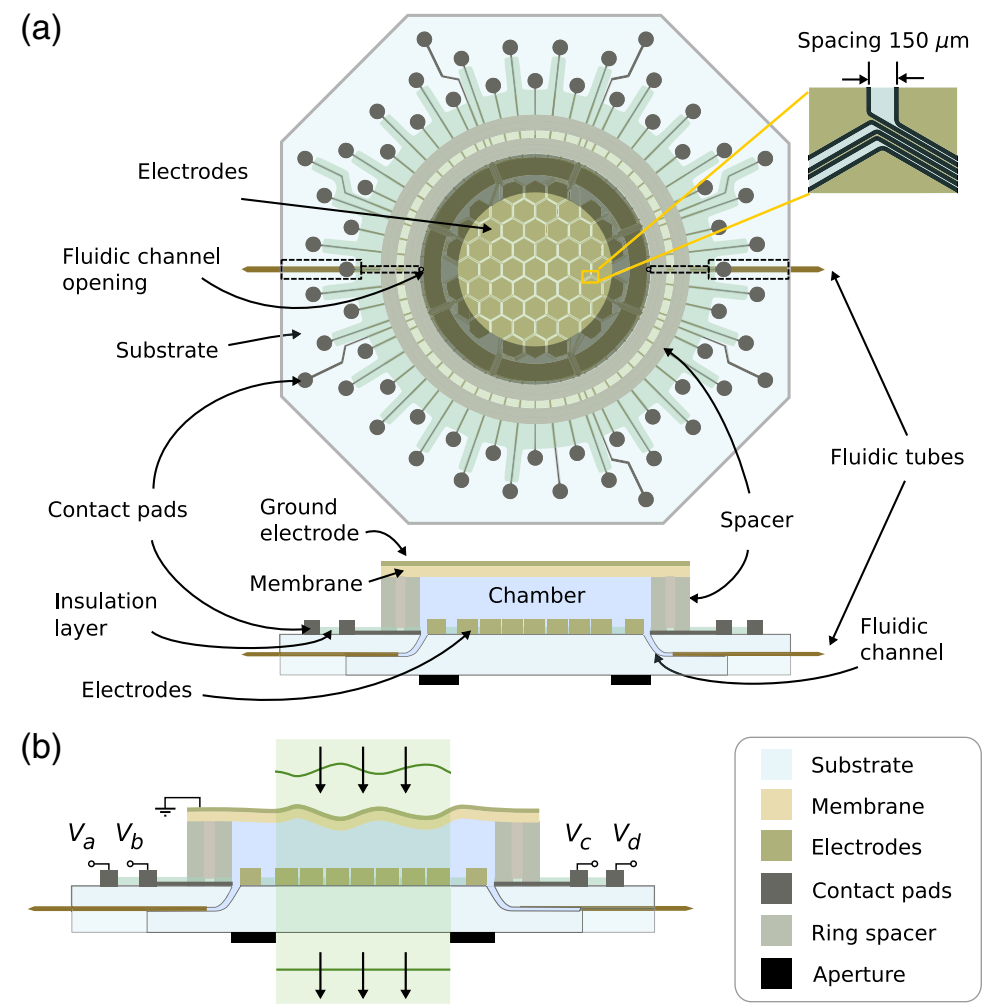

Fig. 2 Concept of the developed optofluidic wavefront modulator. (a) Schematics of the top and cross-sectional views of the DPP. The inset of the top view shows the electrical interconnect lines between the electrodes. (b) Working principle of the DPP: a differential voltage between the grounded membrane and individual electrodes induces an electrostatic force that deforms the DPP's membrane and results in a voltage dependent modulation of optical path.

incompressibility of the liquid and sealing of the chamber volume, there is a resultant hydromechanical coupling that enables a unique bi-directional push/pull mechanism. Positive displacement of the membrane in one portion results in a negative displacement in the other portions as defined by the conservation of the liquid volume. The phase modulation associated with a membrane deformation $\mathbf{a}_{m}$ is given by

$$
\mathbf{a}=\mathbf{a}_{m}\left(n_{\text {liquid }}-n_{\text {air }}\right),
$$

where $n_{\text {air }}$ and $n_{\text {liquid }}$ are the refractive indices of air and the optical liquid, respectively. The employed liquid has to be non-polar with a large refractive index to maximize the achievable phase modulation. Furthermore, in addition to stability, it is desired to have a low density to minimize the influence of gravity, high permittivity to maximize the electrostatic force, and low absorption in the operating spectral bandwidth. For wavelengths between 450 and $1100 \mathrm{~nm}$, the current DDP has $>85 \%$ transmission, limited mostly by Fresnel losses from uncoated surfaces. The lower cutoff wavelength, on the other hand, is set by the strong absorption in the employed polymeric material as the deformable membrane $\left(\right.$ Polyimide $^{22}$ ).

\section{Design}

One of the main limitations of the optofluidic devices is the effect of the volumetric gravitational force that acts on the encapsulated liquid. ${ }^{23-25}$ In case of DPP, the thin compliant membrane can deform due to this force when the DPP is operated in a configuration where its optical axis is not parallel to gravity [see Fig. 3(a)]. This may result in spurious wavefront errors (most predominantly in the form of additional tilt and coma) that require correction, and thus effectively reduce 
(a)

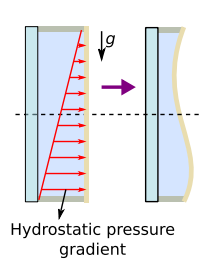

(b)

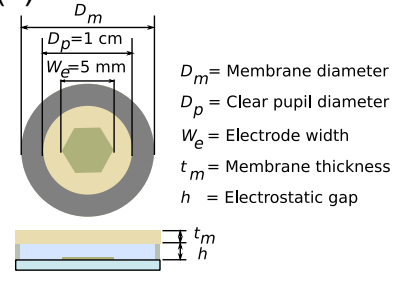

(c)

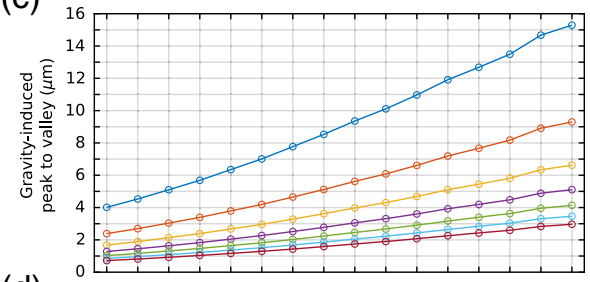

(d)

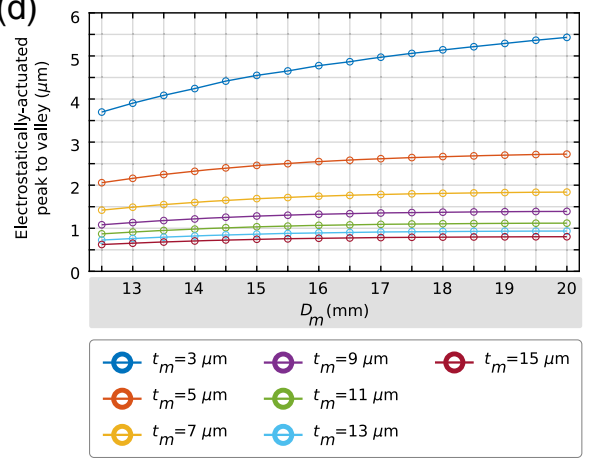

(e)

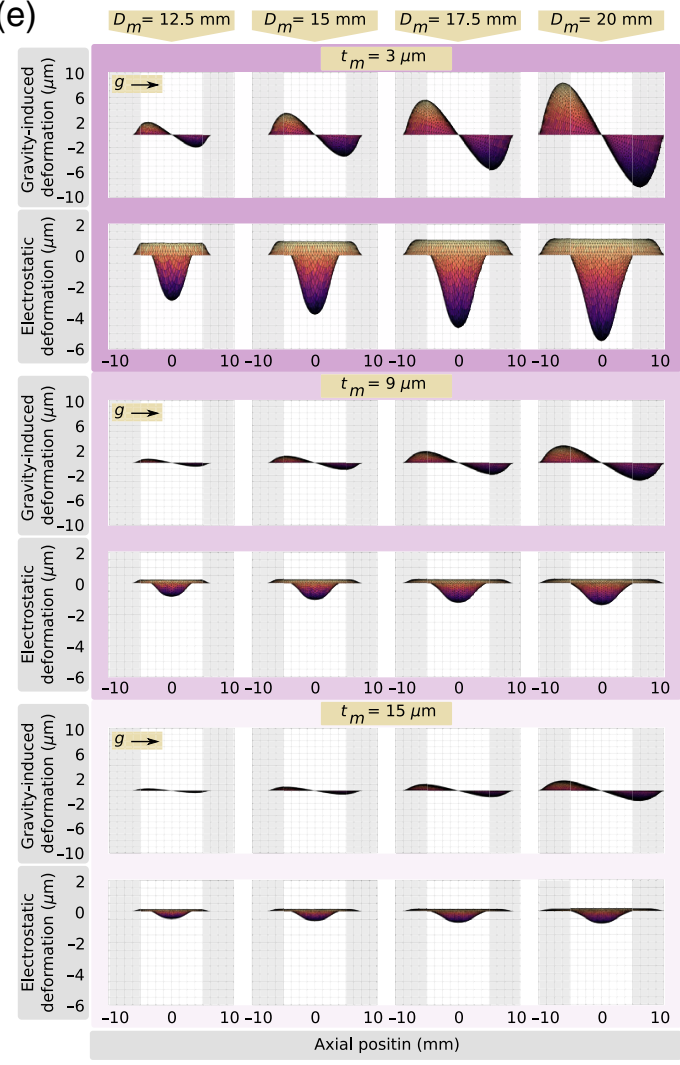

Fig. 3 FE simulation of a simplified multiphysics model of the DPP for investigating an optimal set of parameters for its gravity-neutral performance. (a) Schematic depiction of the influence of gravity-induced hydrostatic pressure on the membrane of DPP. (b) Top and cross-sectional views of the implemented FE model. (c) Peak-to-valley of the gravity-induced deformations of the DPP's membrane with respect to the membrane diameter for different membrane thicknesses, for the worst case of holding DPP vertical. (d) The induced deformations by applying an actuation voltage of $200 \mathrm{~V}$ to the single hexagonal electrode of the FE model with respect to membrane diameter for different membrane thicknesses for $h$ equal to $55 \mu \mathrm{m}$. All the peak-to-valley measurements are performed within the clear pupil of the DPP with fixed diameter $D_{p}$ of $10 \mathrm{~mm}$ and electrode width $W_{e}$ of $5 \mathrm{~mm}$. (e) Side-views of the simulated 3D profiles of the membrane, deformed due to the gravity-induced hydrostatic pressure and electrostatic force, for three different thicknesses $(3,9$, and $15 \mu \mathrm{m})$, and four diameters $(12.5,15,17.5$, and $20 \mathrm{~mm})$. The gray shaded areas on the plots depict the region outside of the $10 \mathrm{~mm} \phi$ clear pupil.

the correction range of the DPP. The main parameters influencing the amount of this unwanted effect on the DPP's membrane deformations and its capability to overcome them are

1. Membrane rigidity $\propto E \sigma_{0} \frac{t_{m}}{D^{\alpha}}$,

where $E$ is the membrane Young's modulus, $\sigma_{0}$ is the pre-stress of the membrane, $t_{m}$ and $D_{m}$ are the membrane thickness and diameter, respectively, and $\alpha$ is a variable that needs to be calculated by solving Poisson's differential equation considering the system boundary conditions. ${ }^{26}$

2. Liquid hydrostatic pressure $\propto \rho l_{h} g$,

where $\rho$ is the density of the liquid, $l_{h}$ is the liquid height parallel to gravity direction, and $g$ is the gravitational acceleration constant.

3. Electrostatic force $\propto \frac{A}{h^{2}} V^{2}$,

where $A$ is the electrode area, $h$ is the electrostatic gap, and $V$ is the applied differential voltage $\left[{ }^{27}\right.$, ch. 6].

The main trade-off in the design of the DPP is between increasing the membrane rigidity to reduce the unwanted gravity effects while maintaining an acceptable stroke. Figure 3(b) shows the schematics of the simplified finite-element analysis (FEA) model of the DPP and the main 
parameters defining its geometry. The hydro-mechanical coupling is ensured by constraining the encapsulated volume between the membrane, the substrate, and the spacer. The effect of gravity is modeled by a pressure gradient on the membrane surface. Figure 3(c) shows the simulated gravity-induced peak-to-valley membrane deformation within the clear pupil area with respect to the membrane diameter $D_{m}$ for different membrane thicknesses $t_{m}$ while keeping the clear pupil diameter fixed at $10 \mathrm{~mm}$. Simulations are performed for the worst case of having DPP's optical axis perpendicular to the gravity direction. By increasing the membrane thickness and reducing its diameter, the membrane's rigidity increases and the gravity-induced deformations diminish significantly. However, increased rigidity also decreases the achievable stroke of the DPP. Figure 3(d) shows the simulated stroke (defined as the achievable peak-to-valley within the pupil area by electrostatic actuation) of the DPP when the single hexagonal electrode is actuated at $200 \mathrm{~V}$ and the electrostatic gap is $55 \mu \mathrm{m}$ (here, gravitational effect is ignored). Compared to the previous design of the DPP ${ }^{13}$ the electrostatic gap is reduced by half to compensate for the required increased membrane rigidity (see the relation between the electrostatic force and gap).

The three groups of 3D plots in Fig. 3(e) show the simulated profile of the deformable membrane for three different thicknesses (3, 9, and $15 \mu \mathrm{m}$ ), where for each thickness the simulation is performed for four membrane diameters $(12.5,15,17.5$, and $20 \mathrm{~mm})$. These are selected examples from the presented results in the line plots of Figs. 3(c) and 3(d), depicting gravity-induced and electrostatically actuated deformations of the membrane. The plots show the side-view of the membrane and the area outside of the clear pupil region is shaded in gray. While enlarging the membrane diameter causes larger gravity-induced deformations and overall electrostatic actuation, it has a diminishing effect for increasing the achievable electrostatically actuated deformations within the clear pupil. A membrane area of larger than the clear pupil (10 $\mathrm{mm} \phi$ in this case) is advantageous for replicating sharp wavefront slopes at the edges of the pupil. However, a membrane diameter of more than $15 \mathrm{~mm}$ significantly increases the unwanted gravity-induced deformations without a considerable added benefit. To achieve a balance between the membrane rigidity and the active stroke, we chose a thickness of $9 \mu \mathrm{m}$ and a diameter of $15 \mathrm{~mm}$ for the membrane of the current DPP implementation. Furthermore, in the final design, the overall area of the electrodes, including outside of the pupil area, is maximized to further increase the available electrostatic force. Table 1 summarizes the detailed properties of the developed DPP.

As shown in Fig 2(b), the DPP has 55 hexagonal electrodes mostly within the clear pupil, and 8 arc-shaped electrodes distributed in the form of a segmented ring outside of the clear pupil. The inner pupil hexagonal electrodes, via their small area and large fill-factor, maximize the degrees of freedom for high spatial frequency wavefront modulation. The arc electrodes outside the aperture are intended for three main purposes: (1) enabling reproduction of sharp slopes at the edges of the wavefronts, (2) increasing the push forces (resulting in the outward deformation of the membrane) inside the pupil by further exploiting the available hydro-mechanical coupling, ${ }^{14}$ and (3) enhancing the electrostatic force needed to compensate for gravity-induced deformations. In total, the electrode layout of the DPP has a fill factor of $82 \%$ within the clear pupil and $75 \%$ over the entire surface of the fluidic chamber.

\section{Implementation}

\subsection{Wafer-Level 3D Micro-Structuring of Glass}

To fill the fluidic chamber with the optical liquid and seal it hermetically afterward, we developed a new method that uses intra-substrate micro-channels that are etched through the glass substrate by 3D selective laser-induced etching (SLE) of fused silica glass. This process uses focused femtosecond laser pulses to locally modify the fused silica substrate into a form that is attacked by a $\mathrm{KOH}$ solution with very high selectivity. ${ }^{32,33}$ By scanning the laser spot, complex 3D structures can be engraved into the substrate. As opposed to the discrete fluidic ports we previously used, ${ }^{13,16}$ this new interfacing method does not increase the device thickness [Fig. 2(a)], allowing cascading of multiple devices within close proximity.

The drawing in Fig. 4(a) and its inset depict an SLE-processed 4 in. wafer bearing four DPP chips and the cross-sectional and bottom views of one of the fluidic channels. The octagonal 
Rajaeipour et al.: Seventh-order wavefront modulation with a gravity-neutral optofluidic deformable...

Table 1 List of properties for the developed optofluidic DPP in this study.

\begin{tabular}{|c|c|}
\hline \multicolumn{2}{|l|}{ General properties } \\
\hline Actuation principle & Electrostatic \\
\hline Modulation type & Continuous \\
\hline Number of electrodes & 63 \\
\hline Electrode count across pupil & 7 \\
\hline Driving voltage & $0-350 \mathrm{~V}$ \\
\hline Geometries & Value \\
\hline Clear pupil diameter & $10 \mathrm{~mm}$ \\
\hline Fluidic chamber diameter & $15 \mathrm{~mm}$ \\
\hline Electrode area diameter & $14.5 \mathrm{~mm}$ \\
\hline Electrostatic gap & $55 \mu \mathrm{m}$ \\
\hline Membrane thickness & $9 \mu \mathrm{m}$ \\
\hline Substrate thickness & $800 \mu \mathrm{m}$ \\
\hline Chip length & $33 \mathrm{~mm}$ \\
\hline Use & Material \\
\hline Deformable membrane & Polyimide $(E=9.8 \mathrm{GPa})^{22}$ \\
\hline Substrate & Fused Silica \\
\hline Transparent electrodes & Indium tin oxide $(\mathrm{ITO})^{28}$ \\
\hline Isolating dielectric & Parylene $\mathrm{C}^{29}$ \\
\hline Optical liquid & Tridecane $(n=1.4256)^{30}$ \\
\hline Contact pads & Platinum \\
\hline Ring spacer & Ordyl $\left.\right|^{31}$ \\
\hline Fluidic tubes & Brass \\
\hline
\end{tabular}

dashed lines depict the borders of the DPPs, which require two fluidic channels each, one for filling the liquid and another one for venting during the filling process. The dot-dashed lines in the detailed view of the fluidic channel show the center of the channel and the dotted line shows the dicing path where the DPP chip is singulated. The alignment marks for further lithography steps are also formed by femtosecond laser engraving, but they are buried inside the wafer such that they are protected during the etching process. The engraved channels are etched in a $\mathrm{KOH}$ bath (concentration $8 \mathrm{~mol} / 1$, at $80^{\circ} \mathrm{C}$ ) for about $60 \mathrm{~h}$. The image in Fig 4(a) shows the glass wafer after the wet-etching process. The fluidic channels are opened and the alignment marks are remained untouched.

\subsection{DPP Chip Manufacturing and Assembly}

The manufacturing steps of DPP after micro-structuring of the fluidic channels are shown in Fig. 4. The main processes performed on the micro-structured fused silica wafers are as following [Fig. 4(b)]:

1. About $100 \mathrm{~nm}$ of platinum is patterned by a lift-off process to create the contact pads and electrical tracks. 


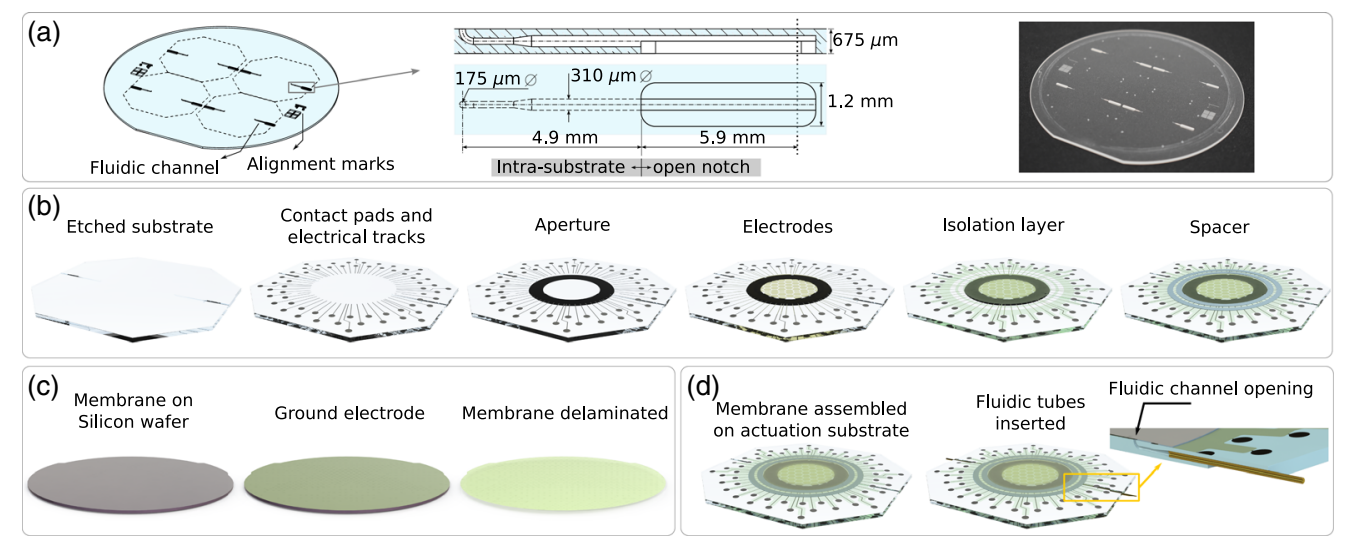

Fig. 4 Fabrication steps of the DPP's actuation substrate and deformable membrane. (a) Waferlevel 3D micro-structuring of glass for fluidic interfacing of the DPP. The drawing shows the pattern that is engraved using an ultra-short pulsed laser on a $800-\mu \mathrm{m}$-thick 4 in. Fused Silica wafer. The inset shows the detailed view of one of the micro-fluidic channels. The photo shows the Fused Silica wafer after the SLE process. (b) For fabrication of the actuation substrate: the wafer with the structured micro-fluidic channels is employed. Metalization processes are performed for patterning the contact pads and electrical tracks, the opaque aperture on the bottom surface and the transparent electrodes. Parylene $\mathrm{C}$ is coated as an isolation layer to protect the small spacing between the electrodes and the tracks from electrical break-down. The spacer is laminated and structured. At this stage the processed wafer is diced into 4 octagonal actuation substrates. (c) For the membrane: the liquid Polyimide is spin-coated on a $100-\mathrm{mm}$ silicon oxide wafer and thermally cured, its surface is coated by ITO, and the membrane is delaminated by peeling. (d) For assembly the channel on the spacer is primed with epoxy and the substrate is placed on the stretched membrane. Brass tubes are inserted and glued into the micro-fluidic channels.

2. The aperture that defines the clear pupil area is patterned on the backside of the glass substrate and is made of multiple metal layers, including platinum, iridium, and iridium oxide to create a completely opaque structure. An additional coating of silicon oxide protects this layer from physical damage.

3. About $90 \mathrm{~nm}$ of indium tin oxide (ITO) is patterned to create the transparent electrode array and the interlaced electrical tracks.

4. About $\sim 3.5 \mu \mathrm{m}$ of parylene $\mathrm{C}$ is coated on the top surface of the substrate to provide electrical isolation between the electrodes and the tracks. For choosing the sufficient thickness of the parylene, it is necessary to consider the maximum driving voltage and the minimum distance between the electrodes and tracks to prevent dielectric failure.

5. Finally a 55- $\mu \mathrm{m}$-thick layer of Ordyl (a dry film photoresist) is laminated on top surface of the substrate and is patterned by photolithography to provide the spacer structure to support the membrane. The Ordyl structure also forms a channel that assists in the assembly by guiding the epoxy application.

6. The DPP chips are diced into an octagonal form.

The fabrication steps for the 9- $\mu$ m-thick deformable membrane made of Polyimide are as follows [Fig. 4(c)]:

1. The liquid Polyimide varnish, UPIA-ST, ${ }^{22}$ is spin-coated on a 4 in. silicon oxide wafer, followed by thermal imidization of the Polyimide.

2. About $50 \mathrm{~nm}$ of ITO is sputtered on the top surface of the cured Polymide to make it conductive.

3. The Polyimide membrane is manually delaminated by peeling.

Figure 4(d) shows the assembly steps of the DPP. Prior to assembly on the actuation substrate, the membrane is secured and stretched on a custom machined stretcher frame. After 

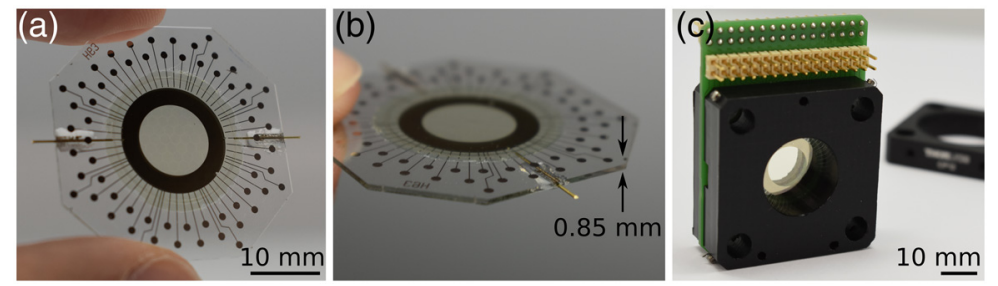

Fig. 5 Fabricated DPP. (a) Front and side views. The black ring defines the DPP's clear pupil with a diameter of $10 \mathrm{~mm}$. (b) DPP in its $30-\mathrm{mm}$ cage system compatible housing with electrical interfacing.

dispensing epoxy in the channel within the Ordyl spacer, the actuation substrate is placed on the stretched membrane, and the whole assembly is cured. Next, two brass tubes (outer diameter $300 \mu \mathrm{m}$, inner diameter $100 \mu \mathrm{m}$ ) are inserted in the fluidic channels within the actuation substrate. The open notches of each fluidic channel are primed with epoxy to fix and seal the brass tubes. Subsequently, the fluidic chamber is filled with a non-polar liquid (tridecane ${ }^{30}$ ). For this, the brass tubes are interfaced with polyether ether ketone (PEEK) micro-tubes and the filling is performed under an interferometer for a precise and real-time control on the amount of liquid inside the chamber. Finally, the brass tubes are cut and crimped to completely seal the fluidic chamber. The front and side views of the fabricated DPP are shown in Fig. 5(a).

For convenient integration of the DPP into optical systems, we developed a housing compatible with the widely used 30-mm optomechanical cage system. Figure 5(b) shows the DPP in this housing. For electrical interfacing to the contact pads, the printed circuit board with 64 pogo pins (63 pins for actuation channels and 1 pin for ground connection to the membrane top surface) is used.

\section{Characterization and Control}

For quantitative characterization of the DPP's wavefront shaping performance, we employed a custom Mach-Zehnder interferometer. Like a lens with similar dimensions, DPP can be inserted in the optical path of the interferometer's measurement arm without requiring additional beam folding optics. The interferograms are captured using a camera (XIMEA MC-124MG-SY), and a spatial-carrier fringe-pattern analysis technique is used for extracting the quantitative phase data from the captured interferograms. Details of this implementation can be found elsewhere. ${ }^{34}$ For convenience in wavefront representation, the measured phase data are fitted to Zernike polynomials using a total of 136 modes (up to 15th radial order) for a precise fit. The Zernike polynomials are expressed by the OSA standard indexing scheme without normalization of the coefficients. ${ }^{35}$ Throughout this study, unless specifically mentioned, the effect of piston, tip, and tilt modes are ignored as they are not considered as optical aberrations. When measurement of the tip and tilt is required, we employ a Shack-Hartmann wavefront sensor (Thorlabs WFS405C). This is to avoid any confusion between the tilt induced by DPP and the tilt added for the spatial-carrier fringe-pattern analysis technique.

We have previously formulated an optimal control problem for calculating the driving signals of DPP based on its empirical model in open-loop, which also takes into account the system boundary conditions such as the minimum and maximum applicable voltages. In this approach, the input is the target wavefront shape described by its Zernike coefficients and the output are the control signals of all the DPP electrodes. We employ the same control algorithm in the current study and the details of its implementation can be found elsewhere. ${ }^{36}$ This approach is based on two main requirements. (1) Existence of a linear relationship between the signals applied to each of DPP's electrodes and its surface profile, and (2) the influence functions (the imprints of singular electrodes with a unit control signal) of all electrodes being linearly independent. If these two requirements are satisfied, any arbitrary surface profile can be replicated as a linear superposition of the individual influence functions. 
Rajaeipour et al.: Seventh-order wavefront modulation with a gravity-neutral optofluidic deformable...

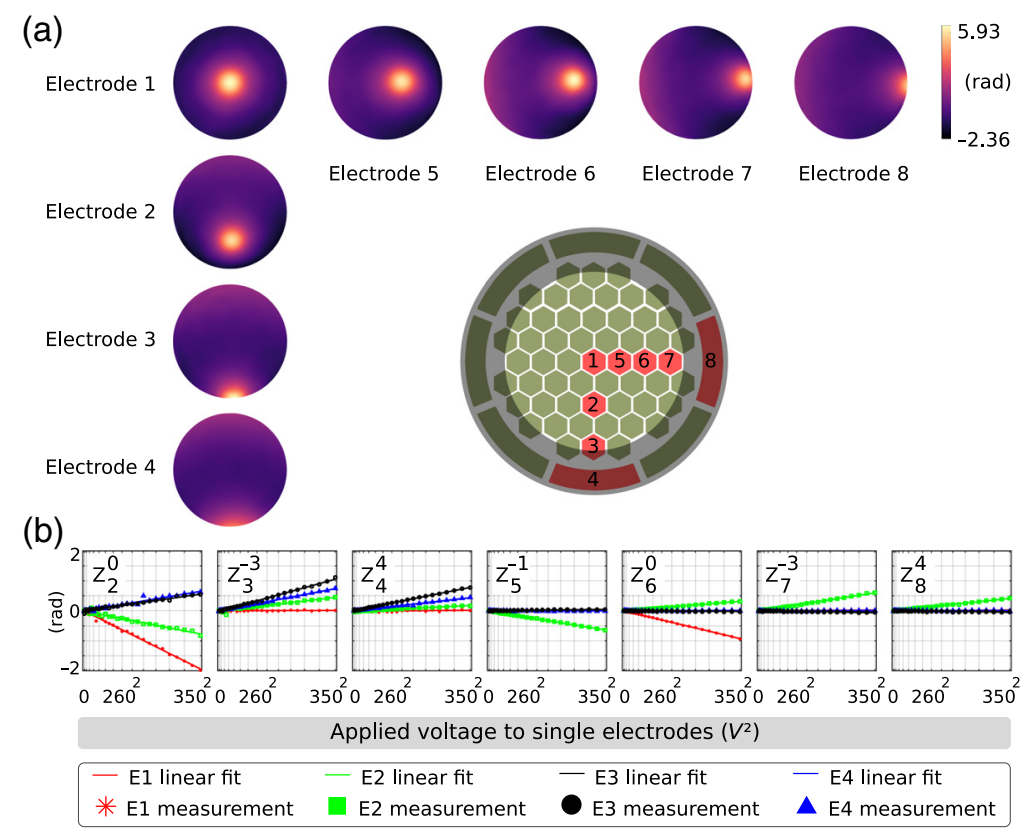

Fig. 6 Investigating the response linearity of the DPP. (a) The profiles of the measured wavefronts by actuating eight distinct electrodes of DPP at $350 \mathrm{~V}$. The selected electrodes are colored in red in the depicted electrode layout and the area outside of the clear pupil is shaded in gray. (b) Plots depicting the relation of selected Zernike modes from second to eighth order, with respect to the square of the applied voltage signal from 0 to $350 \mathrm{~V}$ at steps of $12.5 \mathrm{~V}$ on a single electrode. Each color/shape corresponds to a separate electrode (E1 to E4).

Figure 6(a) shows the influence of eight individual electrodes of the DPP at 350-V drive voltage, where each electrode is placed at a different radial position. Positions of the selected electrodes are highlighted in red in the drawn electrode layout of the DPP and the area outside of the clear pupil is shaded in gray. The distinctive and localized influence of individual electrodes is crucial for successful implementation of its control algorithm. One of the main advantages of the DPP compared to the other non LC-SLM refractive wavefront modulators is this capability of having a 2D control over the phase modulation within its clear pupil with high spatial frequency. To demonstrate the linear relationship between electrode control signals and their influences on the membrane deformation, we swept driving voltage of individual electrodes from 0 to $350 \mathrm{~V}$ with steps of $12.5 \mathrm{~V}$, and measured the resulting wavefront in terms of Zernike coefficients. Figure 6(b) shows the results of these measurements for seven exemplified Zernike modes from the second order to eighth order. We evaluated the linear fit of the individual Zernike coefficients for each electrode with respect to different powers of voltage. The shapes on the plots depict the measured data-points and the lines are the linear fits. The closest linear fit of the Zernike coefficients could be found for the voltage to the power of 2 . As a result, we chose the control signal $c$ for electrode $l$ to be equal to

$$
c_{l}=v_{l}^{2}
$$

The influence function of each electrode is composed of the slope of the linear fits [e.g. the seven slope values for the shown seven Zernike modes as shown in the subplots of Fig. 6(b)]. We use two measurement points for calculating the influence functions of each electrode; one at $V_{\text {min }}=0 \mathrm{~V}$ and one at $V_{\max }=350 \mathrm{~V}$. To validate the second requirement for open-loop control, we calculated the rank of the influence matrix $\mathbf{B}$, and confirmed that it is equal to the number of electrodes of the DPP. Therefore, all the vector columns of the influence matrix (the individual influence functions) are linearly independent from each other. 


\section{Experimental Results}

\subsection{Initial Flatness and Active Stroke}

Figure 7(a) shows the Zernike decomposition of the initial wavefront error of the DPP in both horizontal and vertical operation modes, with all electrodes set to $0 \mathrm{~V}$, and with the device brought to an active best flat by applying the required wavefront correction. The root-meansquare (RMS) of the wavefront error is reduced from $0.447 \lambda$ to $0.016 \lambda$ for the horizontal mode and from $0.489 \lambda$ to $0.017 \lambda$ for the vertical mode. The wavefront error for both orientations of the DPP results in an approximate Strehl ratio of 0.99 , which is well below the Maréchal quality criterion. As a result, DPP may be used for diffraction-limited imaging applications independent of its orientation. The initial wavefront error of the DPP is sufficiently small and is always actively compensated without employing a considerable amount of its available stroke for wavefront correction. Figure 7(b) shows the profiles of the corresponding wavefront errors. The visible print-through of the electrodes is the result of the passively induced optical path difference due to the difference between the refractive indices of the transparent electrodes (90-nm-thick sputtered ITO, ${ }^{28} n \approx 1.7$ ) and the optical liquid (tridecane, ${ }^{30} n=1.4256$ ). However, these have a relatively small influence on imaging performance due to the small induced optical path difference and only contributing to very high-order aberration modes. ${ }^{15,16}$ Nevertheless, this influence can still be mitigated by matching the refractive indices; by using an optical liquid with a higher refractive index and by reducing ITO's refractive index by controlling the sputtering process parameters. The dominant aberration mode for the all off condition is defocus that results from DPP's fluidic chamber being slightly overfilled. This overfilling is intentional and helps with boosting the available push/pull mechanism due to the previously mentioned hydro-mechanical coupling. The remaining aberration modes, excluding the gravity-induced portion of Coma, arise from the inherent initial deformations of the DPP's membrane due to the imperfections during its assembly.

(a)

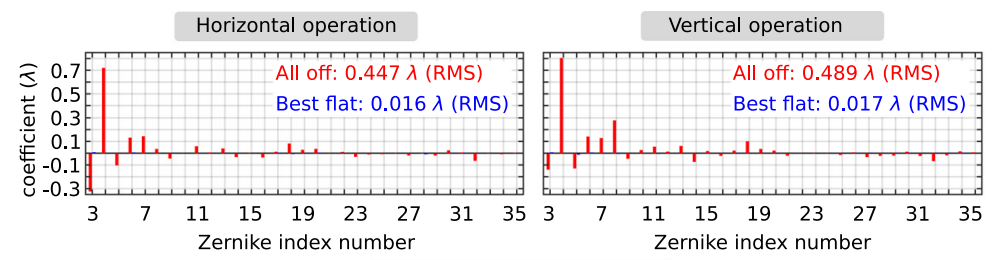

(b)

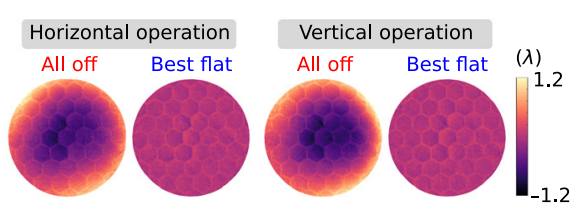

(c)

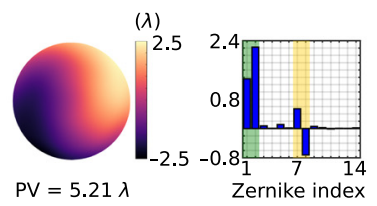

(d)

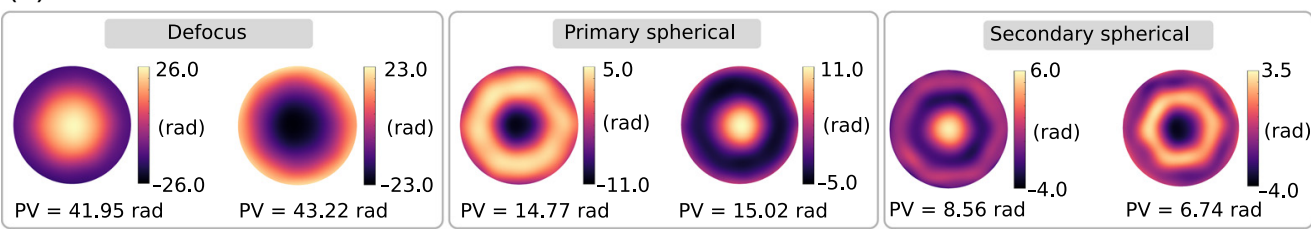

Fig. 7 Initial wavefront error of DPP for vertical and horizontal operation modes in all off and best flat conditions represented by the (a) Zernike decomposition and (b) profiles of the corresponding measured wavefronts. (c) Gravity-induced wavefront error of the DPP after subtracting the initial error of its deformable membrane. The tilt and coma modes are shaded in green and yellow, respectively. (d) Profiles of the measured maximum positive and negative defocus $Z_{2}^{0}$, primary spherical $Z_{4}^{0}$, and secondary spherical $Z_{6}^{0}$ aberration modes to investigate the active stroke of the DPP. 
To measure the pure gravity-induced wavefront error of the DPP, we employ a ShackHartmann wavefront sensor. All the components for this setup including the laser, DPP, and the sensor are assembled in-line on a rotational mount. While DPP is horizontal, we calibrate the sensor and subtract the measured wavefront to ignore the effect of all other sources of aberrations including DPP's initial wavefront error. Figure 7(c) shows the profile and Zernike decomposition of the wavefront error measured by rotating the DPP by $90 \mathrm{deg}$. As expected, the resulting wavefront includes mostly tilt (shaded in green) and coma (shaded in yellow). The peak-to-valley of the gravity-induced wavefront at a wavelength of $533 \mathrm{~nm}$ corresponds to an optical path difference of $2.78 \mu \mathrm{m}$ and depicts a $18 \%$ deviation from the estimated value from the FEA simulations. This variation arises from the differences in the stiffness of the experimentally assembled membrane and its simulated model.

The maximum stroke of the DPP after compensation for its initial wavefront error is investigated by generating opposite amplitudes of defocus $Z_{2}^{0}$, primary spherical $Z_{4}^{0}$, and secondary spherical $Z_{6}^{0}$ aberration modes. Figure 7 (d) shows the profiles of the measured maximum positive and negative amplitudes of these modes. For replicating negative defocus, the center electrodes of DPP are driven to generate an electrostatic pulling force at the center resulting in a maximum peak-to-valley of $43.22 \mathrm{rad}$. For replicating positive defocus, mainly the electrodes outside of the clear pupil are actuated to pull from the outer region of the membrane by electrostatic force and subsequently, due to the displacement of the incompressible liquid, the center of the membrane is pushed upward by the resultant force of the hydro-static pressure. This effect results in a maximum positive defocus of 41.95 rad. Furthermore, DPP is capable of correcting about $14.8 \mathrm{rad}$ of primary and 7.7 rad of secondary spherical aberration modes, in each positive and negative directions, which are particularly important for microscopy applications.

\subsection{Dynamic Behavior}

We evaluated the dynamic behavior of the DPP considering three aspects: hysteresis, creep, and settling time. The electrostatic actuators, while being operated outside of the pull-in regime, are known to be free from hysteresis in their voltage versus displacement behavior. We measured the displacement of the centermost point on the DPP's deformable membrane by applying an increasing and decreasing voltage sweep to the seven middle electrodes of the DPP. In Fig. 8(a), the displacement of the corresponding point is plotted versus the voltage that is applied to the electrodes colored in red. The blue asterisks show forward actuation sweep (steps of $12.5 \mathrm{~V}$ ) from an initial condition with all electrodes at $0 \mathrm{~V}$. The red squares show the backward actuation sweep from an initial condition with the seven corresponding electrodes at $350 \mathrm{~V}$ (all remaining electrodes are at $0 \mathrm{~V}$ ). The symmetry of the forward and the backward sweep indicates a hysteresis-free behavior for the DPP, which significantly simplifies the control algorithm for its open-loop operation. The profiles in Fig. 8(a) show the measured wavefront at the depicted voltage values.

Figure 8(b) shows the results of the performed measurement for investigating the creep behavior of the DPP. For this, we started from the all off condition and applied a random set of voltages to all the DPP electrodes. The applied voltages were constantly held for $20 \mathrm{~min}$. Next, all the channels were set to $0 \mathrm{~V}$ for $2 \mathrm{~min}$, then applied the same set of voltages for $5 \mathrm{~min}$, and finally, all channel were set to off. The resulting wavefronts were recorded every $30 \mathrm{~s}$ and Fig. 8(b) shows their peak-to-valley and RMS. The resulting values are constant within the experimental errors that demonstrate lack of creep for DPP. This is specifically essential for imaging applications were long exposure times are required.

Finally, settling time of the DPP for going from all off condition to another steady-state condition where its electrodes are actuated is measured. For this, the membrane's motion is monitored by focusing the resulting interferogram from the employed interferometer on a photo-detector (Thorlabs PDA100A2). We start with the all off condition and subsequently apply the required set of voltages to replicate a defocus shape with peak to valley of $30 \mathrm{rad}$. Figure 8(c) shows the resulting output of the photo-detector captured using an oscilloscope. The red solid line corresponds to the time when the actuation signals are applied and the dashed line shows the time when membranes motion stops demonstrating a $0 \%$ to $100 \%$ settling time of $\sim 160 \mathrm{~ms}$. It worth emphasizing that the DPP is an over-damped system and the captured fluctuations are the 
(a)
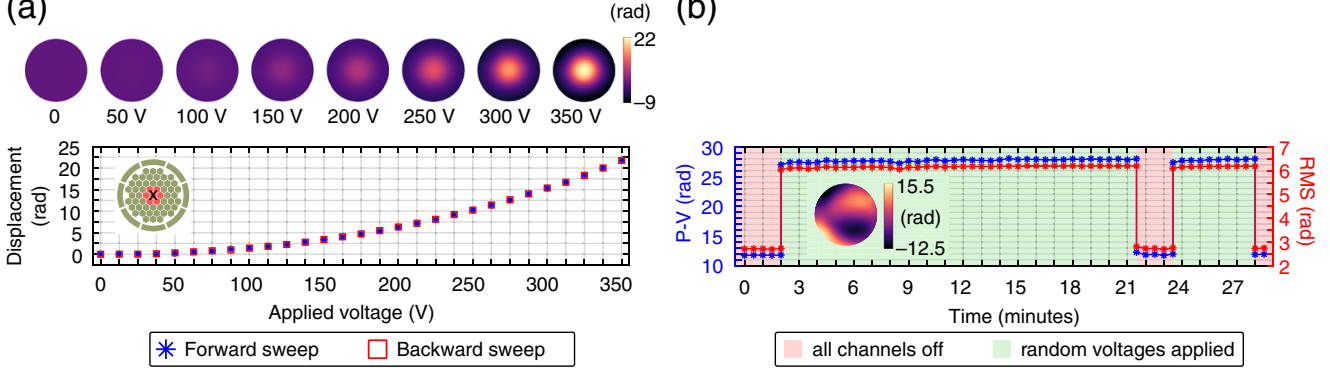

(c)

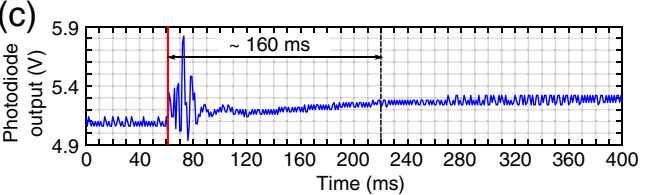

Fig. 8 Dynamic characteristics of DPP. (a) Hysteresis analysis by measuring the displacement of the pupil centermost point in a forward and backward sweep of the applied voltage to the seven middle electrodes of DPP (colored in red). (b) Creep test by configuring the DPP's membrane profile to a random shape and holding the applied voltage for extended periods of time. (c) Monitoring the motion of the DPP's membrane for measuring its $0 \%$ to $100 \%$ settling time by focusing the resulting interferogram on a photo-detector and capturing the output on an oscilloscope.

result of intensity variations in the focused interferogram. It can be seen that the membrane's motion is consisted of a fast period of $30 \mathrm{~ms}$ where the majority of the deformation happens, followed by a damped slower period where the membrane gradually comes to the steady state. While the response time of the DPP compared to existing high-bandwidth DMs may be a limiting factor for high frequency applications, it is sufficient for applications where the aberrations are either static or changing slowly.

\subsection{Zernike Mode Replication}

The wavefront shaping performance of the DPP is investigated by replicating a series of Zernike mode shapes. The replication quality is quantitatively expressed using three figures of merit; Zernike coefficient amplitude of the replicated wavefronts, the RMS error between the obtained profile and their corresponding ideal shape, and the Purity of the replicated modes. Purity is a unitless quantity between 0 and 1 ( 1 being perfect Zernike mode replication, where contribution of all unwanted modes is zero) for quantifying the fidelity of reproducing Zernike modes and is defined as

$$
P_{k}=\frac{a_{k}}{\sqrt{\sum_{i=0}^{M_{f}} a_{i}^{2}}}
$$

where $a_{k}$ corresponds to the coefficient of the target mode $k, i$ is the Zernike index number, and $M_{f}$ is the number of modes used for wavefront representation (here, $M_{f}=136$ ).

To examine the DPP performance for replicating arbitrary wavefront profiles, we reproduce the Zernike mode shapes up to the eighth radial order. The validity of the response model of DPP, as explained in Sec. 5, ensures that if these modes can be successfully reproduced, any of their linear combination (and subsequently any arbitrary wavefront) can also be reproduced. Furthermore, reproduction of orthogonal set of basis functions, such as Zernike modes, is essential for a successful implementation of sensorless wavefront estimation algorithms for AO systems. ${ }^{37}$ Figure 9 shows the coefficient amplitude, RMS error and Purity of replication of sixteen selected Zernike modes from second to eighth radial order. The control signals for replicating all the Zernike modes are calculated in open-loop. 


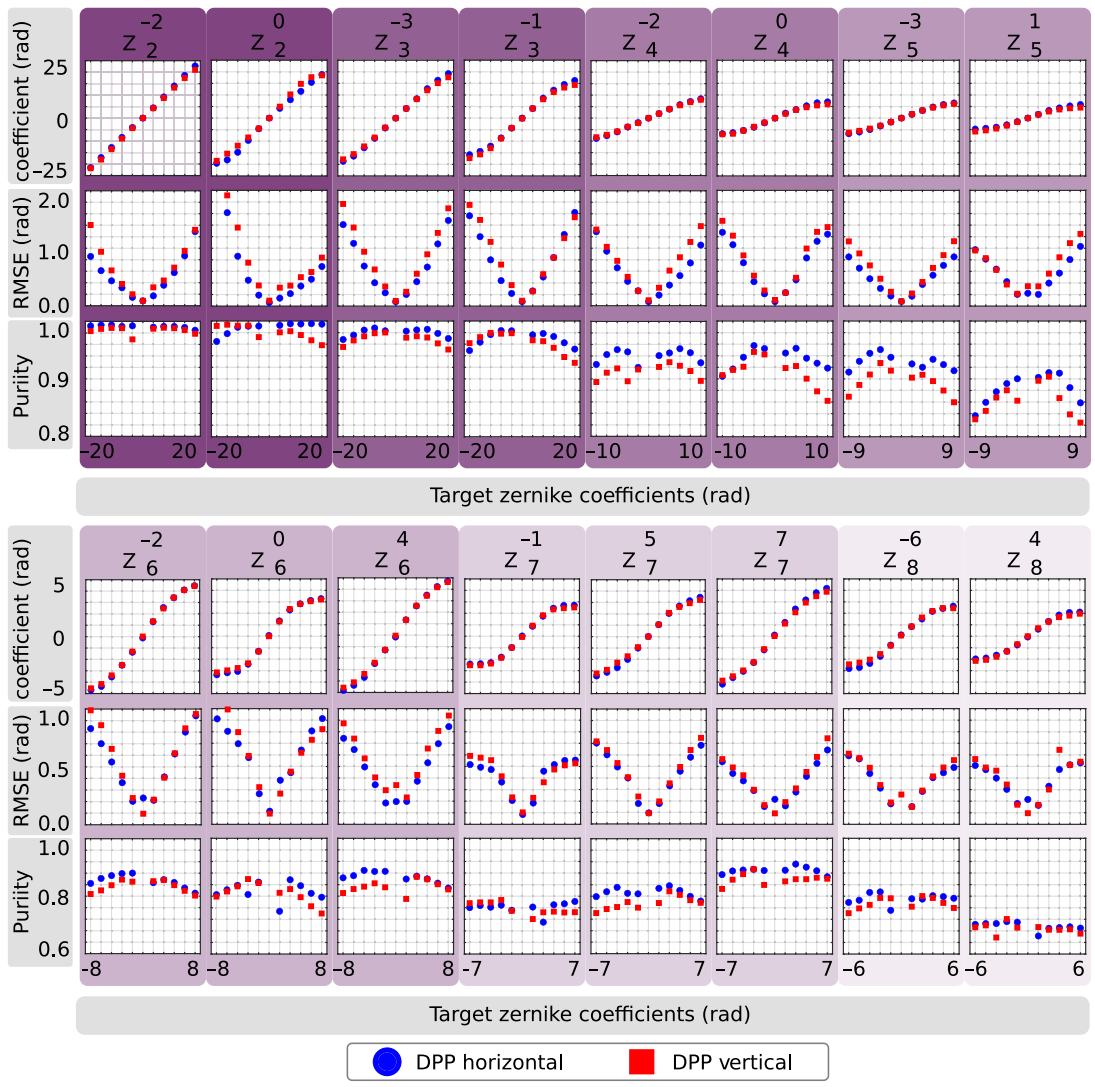

Fig. 9 Experimental characterization results for performance of DPP for replicating up to eighth radial order of Zernike modes while having vertical and horizontal orientations. The plots depict the coefficient amplitude of the replicated modes, their corresponding RMS error and Purity with respect to the target amplitudes of 16 different Zernike modes. The control signals for replicating all the modes are calculated in open-loop using an optimization-based control algorithm described elsewhere. ${ }^{36}$

The Purity of the corresponding amplitudes remain above 0.8 for up to sixth radial order, above 0.7 for seventh radial order, and above 0.6 for eighth radial order. Considering the relatively lower quality of the replicated eighth-order modes, for the current design of the DPP with 63 electrodes, we chose the seventh order as the maximum applicable spatial frequency for wavefront modulation in AO applications. Figure 10 shows the overview of all experimentally replicated Zernike modes of DPP for both vertical and horizontal operation modes. The wavefront profiles correspond to the maximum positive coefficient amplitudes of the replicated Zernike modes. The modulation peak-to-valley at the highest order (seventh order) remains greater than a wavelength. This is the highest spatial frequency wavefront shaping ever achieved with a single refractive wavefront modulator that is not a LC-SLM. Furthermore, by comparing the performance of DPP with and without the gravity effect, it can be seen that the quality of wavefront replication in presence and absence of gravity is nearly identical, making DPP as an optofluidic device suitable for both horizontal and vertical operation modes.

To boost both the amplitude and fidelity of the wavefront modulation, it is possible to employ multiple DPPs in a cascaded configuration. ${ }^{16}$ Due to the significantly small foot-print of DPP and its transmissive nature, two or more DPPs may be integrated serially within the optical path without an excessive increase in the system complexity. In such a manner, one DPP can be configured for large-amplitude low-spatial frequency wavefront modulation, and another one for small-amplitude and high spatial frequency wavefront modulation. 


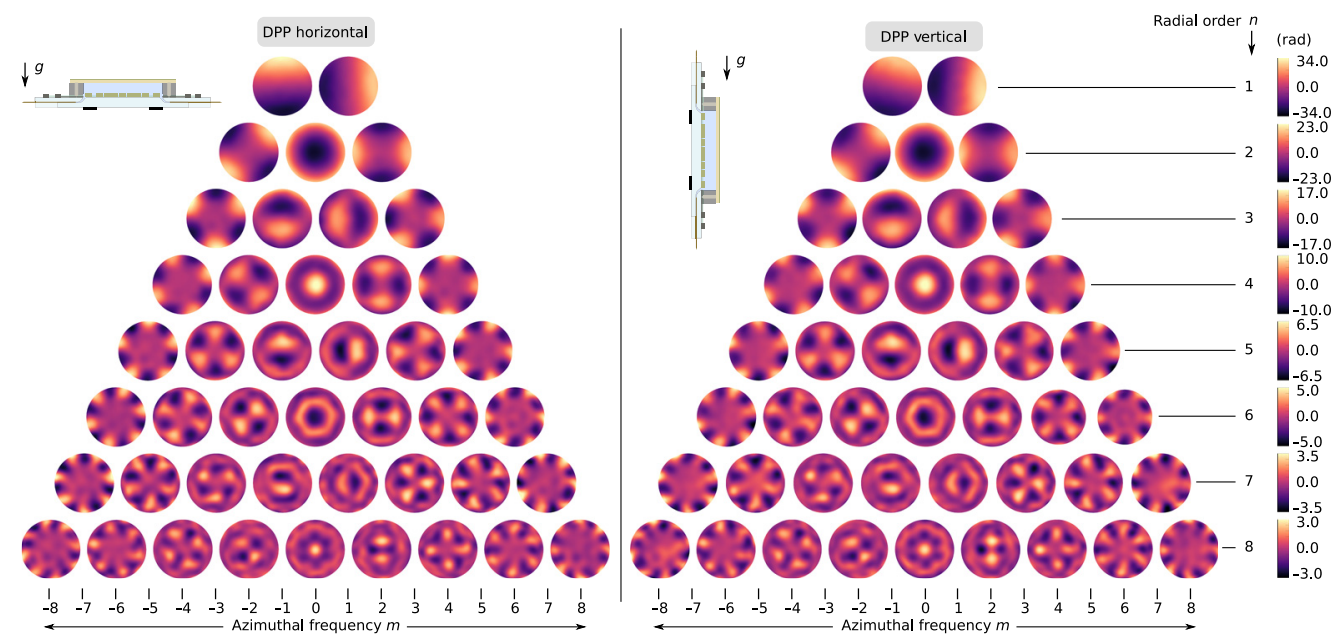

Fig. 10 Overview of the experimentally replicated Zernike modes up to eighth order in open-loop using DPP in horizontal and vertical operation modes. The depicted wavefronts correspond to the maximum positive amplitudes of the Zernike modes that are applicable with the current DPP.

\section{Conclusion}

We presented design and implementation of an optofluidic refractive wavefront modulator with diffraction-limited initial wavefront error and capable of replicating up to seventh radial order of Zernike modes. Compared to other existing refractive solutions such as LC-SLMs and adaptive lenses, it is polarization independent, free from diffraction losses, and offers a scalability similar to continuous face-sheet DMs. Furthermore, unlike majority of optofluidic devices, it was shown that the developed modulator can be operated in both vertical and horizontal orientations without considerable influence of the unwanted gravitational force. With a miniaturized foot-print and transmissive working mode, it enables significantly simplified integration into optical setups without major disturbance or folding the optical path.

\section{Acknowledgments}

This project has received funding from the German Federal Ministry for Economic Affairs and Energy (BMWi) and the European Social Fund (ESF) (Project DPAO, Grant No. 03EFOBW211). Disclosures: The authors declare no conflicts of interest.

\section{References}

1. R. K. Tyson, Principles of Adaptive Optics, CRC Press (2015).

2. F. Roddier, Adaptive Optics in Astronomy, Cambridge University Press (1999).

3. M. J. Booth, "Adaptive optical microscopy: the ongoing quest for a perfect image," Light: Sci. Appl. 3(4), e165 (2014).

4. R. Horstmeyer, H. Ruan, and C. Yang, "Guidestar-assisted wavefront-shaping methods for focusing light into biological tissue," Nat. Photonics 9(9), 563-571 (2015).

5. J. Kubby, S. Gigan, and M. Cui, Wavefront Shaping for Biomedical Imaging, Cambridge University Press (2019).

6. S. Marcos et al., "Vision science and adaptive optics, the state of the field," Vision Res. 132, 3-33 (2017).

7. E. Akyol et al., "Adaptive optics: principles and applications in ophthalmology," Eye 35, 244-264 (2020).

8. R. K. Tyson, "Bit-error rate for free-space adaptive optics laser communications," J. Opt. Soc. Am. A 19(4), 753-758 (2002).

9. P. S. Salter and M. J. Booth, "Adaptive optics in laser processing," Light: Sci. Appl. 8(1), $1-16$ (2019). 
Rajaeipour et al.: Seventh-order wavefront modulation with a gravity-neutral optofluidic deformable...

10. Z. Kam et al., "Modelling the application of adaptive optics to wide-field microscope live imaging," J. Microsc. 226(1), 33-42 (2007).

11. J. Li et al., "Conjugate adaptive optics in widefield microscopy with an extended-source wavefront sensor," Optica 2(8), 682-688 (2015).

12. Q. Li et al., "Woofer-Tweeter adaptive optical structured illumination microscopy," Photonics Res. 5(4), 329-334 (2017).

13. K. Banerjee et al., "Optofluidic adaptive optics," Appl. Opt. 57(22), 6338-6344 (2018).

14. K. Banerjee et al., "Refractive opto-fluidic wavefront modulator with electrostatic push-pull actuation," Proc. SPIE 10886, 108860D (2019).

15. P. Rajaeipour et al., "Fully refractive adaptive optics fluorescence microscope using an optofluidic wavefront modulator," Opt. Express 28(7), 9944-9956 (2020).

16. P. Rajaeipour et al., "Cascading optofluidic phase modulators for performance enhancement in refractive adaptive optics," Adv. Photonics 2(6), 066005 (2020).

17. F. O. Fahrbach et al., "Rapid 3D light-sheet microscopy with a tunable lens," Opt. Express 21(18), 21010-21026 (2013).

18. K. Philipp et al., "Diffraction-limited axial scanning in thick biological tissue with an aberration-correcting adaptive lens," Sci. Rep. 9(1), 1-11 (2019).

19. S. Bonora et al., "Wavefront correction and high-resolution in vivo oct imaging with an objective integrated multi-actuator adaptive lens," Opt. Express 23(17), 21931-21941 (2015).

20. P. Pozzi et al., "Plug-and-play adaptive optics for commercial laser scanning fluorescence microscopes based on an adaptive lens," Opt. Lett. 45(13), 3585-3588 (2020).

21. Z. Zhang, Z. You, and D. Chu, "Fundamentals of phase-only liquid crystal on silicon (LCOS) devices," Light: Sci. Appl. 3(10), e213 (2014).

22. UBE Industries LTD, "UBE UPIA product catalog," https://www.ube.com/upilex/en/ varnish.html (accessed 12-Jan-2021).

23. S. T. Choi et al., "Opto-mechanical analysis of nonlinear elastomer membrane deformation under hydraulic pressure for variable-focus liquid-filled microlenses," Opt. Express 22(5), 6133-6146 (2014).

24. P. Zhao, Ç. Ataman, and H. Zappe, "Gravity-immune liquid-filled tunable lens with reduced spherical aberration,” Appl. Opt. 55(28), 7816-7823 (2016).

25. Z. Lu and L. Cai, "Calibration method for the electrically tunable lens based on shapechanging polymer,' Opt. Express 28(21), 31140-31162 (2020).

26. E. Ventsel, T. Krauthammer, and E. Carrera, "Thin plates and shells: theory, analysis, and applications," Appl. Mech. Rev. 55(4), B72-B73 (2002).

27. S. D. Senturia, Microsystem Design, Springer Science \& Business Media (2007).

28. C. G. Granqvist and A. Hultåker, "Transparent and conducting ito films: new developments and applications," Thin Solid Films 411(1), 1-5 (2002).

29. Para Tech Coating, "Specifications \& properties for parylene C," http://www.parylene.com/ Parylene-Technology/Specifications-Properties (accessed 12-Jan-2021).

30. National Center for Biotechnology Information, "PubChem compound database; CID=12388," https://pubchem.ncbi.nlm.nih.gov/compound/12388 (accessed 12-Jan-2021).

31. Elga Europe s.r.1., "ORDYL SY 300 - product data sheet," https://www.elgaeurope.it/en (accessed 12-Jan-2021).

32. J. Gottmann, M. Hermans, and J. Ortmann, "Microcutting and hollow 3D microstructures in glasses by in-volume selective laser-induced etching (ISLE)," J. Laser Micro Nanoeng. 8(1), 15 (2013).

33. M. Hermans, J. Gottmann, and F. Riedel, "Selective, laser-induced etching of fused silica at high scan-speeds using KOH," J. Laser Micro/Nanoeng. 9(2), 126-131 (2014).

34. P. Rajaeipour et al., "Optimization-based open-loop control of phase modulators for adaptive optics," Proc. SPIE 10886, 108861A (2019).

35. L. N. Thibos et al., "Standards for reporting the optical aberrations of eyes," J. Refract. Surg. 18(5), S652-S660 (2002).

36. P. Rajaeipour et al., "Optimization-based real-time open-loop control of an optofluidic refractive phase modulator,” Appl. Opt. 58(4), 1064-1072 (2019). 
Rajaeipour et al.: Seventh-order wavefront modulation with a gravity-neutral optofluidic deformable...

37. Q. Hu et al., "A universal framework for microscope sensorless adaptive optics: generalized aberration representations," APL Photonics 5(10), 100801 (2020).

Pouya Rajaeipour received his BSc degree in mechanical engineering from Isfahan University of Technology and his MSc degree in microsystems engineering from the University of Freiburg in 2015 and 2017, respectively. He is currently a PhD candidate at the Gisela and Erwin Sick Laboratory of Micro-Optics, the University of Freiburg in Germany. The focus of his research is on refractive $\mathrm{AO}$ systems.

Martin Sauther received his BEng degree in mechanical engineering from BadenWuerttemberg Cooperative State University (DHBW) Loerrach and his MSc degree in microsystems engineering from the University of Freiburg, in 2014 and 2020, respectively. From 2014 to 2017, he worked as project engineer at Stryker Leibinger GbmH in Freiburg. He is currently a R\&D project manager at the Physik Instrumente GmbH in Karlsruhe, Germany.

Kaustubh Banerjee is a researcher at the Chair of Micro-Optics at the University of Freiburg, Germany. His work focuses on developing optofluidic devices with particular applications for AO. He earned his BSc in mechanical engineering at UCLA in 2012 followed by an MSc and $\mathrm{PhD}$ in microsystems engineering at the University of Freiburg in 2014 and 2020 respectively.

Hans Zappe is Gisela and Erwin Sick professor of micro-optics at the University of Freiburg in Germany. He earned his BSc and MSc degrees at MIT in 1983 and a PhD from the University of California, Berkeley, in 1989, all in electrical engineering. He worked at IBM (USA), the Fraunhofer Institute for Applied Solid State Physics (Germany) and the Centre Suisse d'Electronique et de Microtechnique (Switzerland), before joining the Department of Microsystems Engineering at the University of Freiburg in 2000. He was dean of engineering from 2008 to 2010, dean of studies from 2002 to 2008, and currently serves as department chair. $\mathrm{He}$ is a fellow of SPIE, OSA, and IOP.

Çağlar Ataman received his $\mathrm{PhD}$ in electrical engineering in 2008 from Koç University (Istanbul, Turkey), and worked as a post-doctoral researcher at the École Polytechnique Fédérale de Lausanne (EPFL) (Switzerland) between 2008 and 2012. Since 2012, he is a senior scientist and a group leader in the Micro-Optics Laboratory at the University of Freiburg (Germany). His current research focuses on optical microsystems for biomedical imaging and life-science microscopy. 